\title{
Scattering From a Conducting Sphere Embedded in a Semi-Infinite Dissipative Medium
}

\author{
Janis Galejs \\ Contribution from Applied Research Laboratory, Sylvania Electronic Systems, Waltham 54, Mass.
}

(Received March 12, 1962; revised April 18, 1962)

\begin{abstract}
The conducting sphere is embedded in a semi-infinite dissipative medium and is excited by a surface wave or by vertical electric and horizontal magnetic dipoles from the lossless half-space. The sphere acts as a combination of horizontal magnetic and electric dipoles. Its dipole moment depends on the wave incident from the interface of the two media and on the multiple reflections between the sphere and the interface. The transient reflections are calculated after establishing the overall harmonic response of the system.
\end{abstract}

\section{Introduction}

A two-region problem of a conducting sphere in an infinite dissipative medium has been considered by Wait [1951, 1953, 1960a] for an excitation by a timevarying magnetic field, by a coil, which carries an oscillating current, or by dipoles. In this note the above problem is extended to a conducting sphere embedded in a semi-infinite dissipative medium.

Related topies, applicable to the solution of the overall problem, have been discussed in literature. Fields of dipoles above and in lossy media [Sommerfeld, 1949; Stratton, 1941; Norton, 1936, 1937; Baños and Wesley, 1953, 1954; Wait, 1961], the transient propagation in a lossy medium [Ricbards, 1958; Keilson and Row, 1959; Galejs, 1960; Wait, $1960 \mathrm{c}]$, and scattering by a conducting sphere [Stratton, 1941; Wait, 1951, 1953, 1960a] have been considered in detail. Additional references on the subject are listed in the above publications.

This analysis considers the subsurface fields due to a surface wave and due to electric and magnetic dipole excitations in section 2. The fields scattered by the metallic sphere into the lossless medium are computed in several steps. In section 3.1 the fields scattered by the sphere are computed as in an infinite dissipative medium, and the sphere is shown to act as a combination of a horizontal electric and a horizontal magnetic dipole. In a semi-infinite dissipative medium there will be multiple reflections of the fields between the sphere and the interface of the two media. These reflections, which are considered in section 3.2, provide a correction to the equivalent electric and magnetic dipole moments of the sphere. The fields of the equivalent magnetic and electric dipoles, which penetrate into the lossless medium, are computed in section 3.3. After establishing an overall response to harmonic excitations in section 4 , the transient response is calculated by Fourier transform techniques in section 5 .

\section{The Incident Wave}

\subsection{A Plane Surface Wave}

The magnetic field near the surface of region 1 is assumed to be of the form

$$
H_{y 1}^{p l}=H_{0}(\omega) \exp (-i k x)
$$

The field components exhibit a suppressed $e^{i \omega t}$ time dependence. The definition of coordinates is shown in figure 1. In region 2 a compatible solution is of the form

where

$$
H_{y 2}^{p l}=H_{0}(\omega) \exp \left(-i k x-\gamma_{2} z\right),
$$

$$
\begin{aligned}
\gamma_{2} & \approx \sqrt{i \omega \mu_{0} \sigma} \\
k & =\omega \sqrt{\epsilon_{0} \mu_{0}},
\end{aligned}
$$

provided that the displacement currents of medium 2 are negligible $(\omega \epsilon<<\sigma)$. Equations (1) and (2) approximate the variation of the far fields of a dipole over a limited range of $x$. The dimensions of region 3 are much smaller than the free space wavelength $\lambda=2 \pi / k$ and the $x$ variation of the field $H_{y 2}$ is negligible over the sphere of radius $a$. The field at $x \approx 0, z=z_{0}$ is

$$
H_{y 2}^{p l}\left(z_{0}\right)=H_{0}(\omega) \exp \left(-\gamma_{2} z_{0}\right) .
$$

The field variation over the volume of the sphere can be written as

$$
H_{y 2}^{p l}(z)=H_{y 2}^{p l}\left(z_{0}\right) e^{-\gamma_{2}\left(z-z_{0}\right)} \approx H_{y 2}^{p l}\left(z_{0}\right)\left[1-\gamma_{2}\left(z-z_{0}\right)\right]
$$

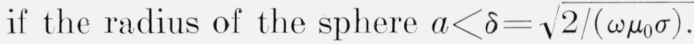

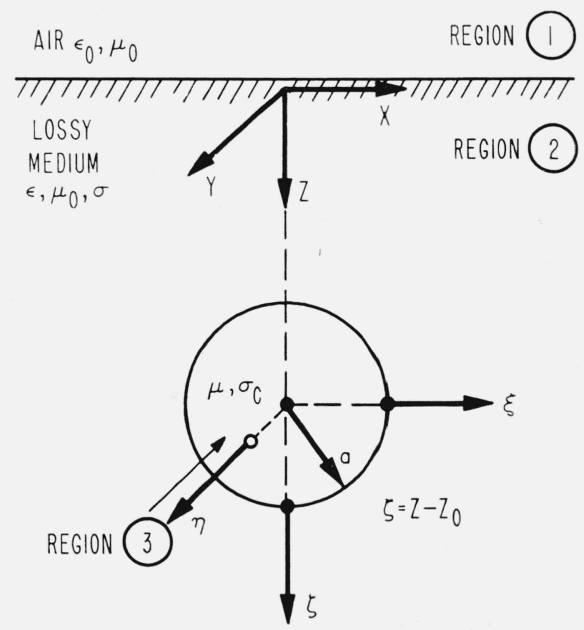

FiguRE 1. The three region geometry. 


\subsection{Near Fields of a Vertical Electric Dipole}

Fields of dipoles that are located on or above a semi-infinite lossy dielectric have been considered by numerous authors [Sommerfeld, 1949; Stratton, 1941; Norton, 1936 and 1937]. The analysis has been usually restricted to fields above or at the surface of the lossy medium. Sources located in the lossy medium are considered by Baños and Wesley [1953 and 1954], while arbitrary coordinates of the source and of the observation point are covered in the analysis of Wait [1961]. The results of Wait [1961] for the horizontal electric dipole will be utilized in the present analysis.

The near fields of a vertical electric dipole (superscript ve) can be obtained from the results which are available for horizontal electric dipoles (superscript he) and from reciprocity relations. It can be shown [Wait, 1960b] that the vertical electric field of a horizontal electric dipole $E_{z}^{\text {he }}$ is related to the radial electric field of a vertical electric dipole $E_{\rho^{\prime}}^{v e}$ by

$$
E_{\rho^{\prime}}^{v e}=E_{z}^{h e} \frac{(I d s)^{h e}}{(I d s)^{v e} \cos \phi}
$$

where the angle $\phi$ is measured from the axis of the horizontal electric dipole and where the coordinates of source and observation point of $E_{\rho^{\prime}}^{r e}$ are interchanged relative to coordinates of $E_{z}^{\text {he }}$. Currents of the $V E$ and $H E$ dipoles flow in $z^{\prime}$ and $x$ directions, where the $x y z$ coordinate system is parallel to the $x^{\prime} y^{\prime} z^{\prime}$ coordinate system. The radial electric field in the lossy medium of a vertical electric dipole of moment $I \dot{d} s$, which is located in the lossless medium, is obtained from (25) and (30) of Wait [1961] and from (7) as

$$
E_{\rho^{\prime}}^{v e}=\frac{I d s \gamma_{2}}{2 \pi \sigma \rho^{\prime 2}}\left(1+i k \rho^{\prime}\right)\left(1+\frac{k^{2}}{\gamma_{2}} z_{s}\right) e^{-i k \rho^{\prime}-\gamma_{2} z^{\prime}},
$$

where the coordinates of the source are $x_{s}, y_{s}, z_{s}$ $\left(z_{s}<0\right)$ and where

$$
\rho^{\prime}=\sqrt{\left(x-x_{s}\right)^{2}+\left(y-y_{s}\right)^{2}}=\sqrt{x^{\prime 2}+y^{\prime 2}} .
$$

If the condition $\left|\gamma_{2} \rho^{\prime}\right|>>1$ is not strictly satisfied, (8) becomes inaccurate and the quasi-static approximations of Wait [1961] should be used instead. The magnetic field in the lossy medium is computed as

$$
H_{\phi^{\prime}}=\frac{-1}{i \omega \mu}\left(\frac{\partial E_{\rho^{\prime}}}{\partial z^{\prime}}-\frac{\partial E_{z^{\prime}}}{\partial \rho^{\prime}}\right) \approx-\frac{1}{i \omega \mu} \frac{\partial E_{\rho^{\prime}}}{\partial z^{\prime}} .
$$

Substituting (8) into (10) and assuming $z_{s}$ to be small,

$$
H_{\phi^{\prime}}^{v e}=\frac{I d s}{2 \pi \rho^{\prime 2}}\left(1+k \rho^{\prime}\right) e^{-i k \rho^{\prime}-\gamma_{2} z^{\prime}} .
$$

The magnetic field at $x \approx 0$ nd $z=z_{0}$ is designated in the notation of the preceding section as

$$
H_{y}^{v e}\left(z_{0}\right)=H_{\phi^{\prime}}^{v e}\left(z_{0}\right)
$$

if $y_{s}=0$ and $x_{s}<0$. Over the volume of the sphere $H_{y}^{v e}(z)$ exhibits the same variation as $H_{y 2}^{p l}(z)$ in (6).

\subsection{Near Fields of a Horizontal Magnetic Dipole}

An expression for the subsurface $H$ field of a horizontal magnetic dipole or of a vertical loop of area $d a$ can be derived with the aid of reciprocity relations from the $H$ field of a horizontal electric dipole. It can be readily shown that

$$
E_{\rho^{\prime}}^{h m}=-i \omega \mu_{0} \sin \phi^{\prime} H_{\phi}^{h e}(\phi=\pi) \frac{(I d a)^{h m}}{(I d s)^{h e}}
$$

where the angles $\phi^{\prime}$ and $\phi$ are measured from the axes of the magnetic and electric dipoles respectively, and where the coordinates of source and observation point of $E_{\rho^{\prime}}^{h m}$ are interchanged relative to coordinates of $H_{\phi}^{\text {he }}$. The superscript $\mathrm{hm}$ refers to the horizontal magnetic dipole and the superscript he refers to the horizontal electric dipole. For negligible heights of the source $z_{s}$, a substitution of $H_{\phi}^{\text {he }}$ of Wait [1961] in (13) and of (13) into (10) results in

$H_{\phi^{\prime}}^{h m}=\frac{I d a}{2 \pi \rho^{\prime 3}}\left[1+\left(i k \rho^{\prime}\right)+\left(i k \rho^{\prime}\right)^{2}\right] e^{-i k \rho^{\prime}-\gamma_{2} z^{\prime}} \sin \phi^{\prime}$

where the angle $\phi^{\prime}$ is measured from the dipole axis. Equation (14) is valid under the same conditions as (8). The magnetic field $H_{\phi^{\prime}}^{h m}$ will be in the $y$-direction at $x \approx 0$ if $y_{s}=0$ and $x_{s}<0$. It will exhibit the $z$-variation of $(6)$ if $z \approx z_{0}$. Equality of (14) with the corresponding field component of a vertical electric (superscript ve) dipole (11) requires for $k \rho^{\prime}$ $<<1$ that

$$
I^{h m} d a \sin \phi^{\prime} / \rho^{\prime}=I^{v e} d s .
$$

\section{The Scattered Field}

\subsection{Scattering by the Sphere in an Infinite Dissipative Medium}

The incident field exhibits an exponential z-variation in (6), (11), or (14), and it may be approximated by a plane wave in the vicinity of the sphere. Scattering of a plane wave by a conducting sphere in an ininite homogeneous medium has been worked out in section 9.25 of Stratton [1941] and can be applied to the present problem without formal difficulties. The scattered fields are expressed as a sum of dipole and higher order multipole modes, but only the dipole modes are significant, if the incident field exhibits only small variations over the volume of the sphere $(a<\delta)$. The $n=1$ terms (dipole modes) of (9.25.4) of Stratton [1941] may be written as

$$
E_{r}^{r}=\frac{i \omega \mu_{0}}{\gamma_{2}} H_{y}\left(z_{0}\right) \frac{3 \exp \left(-\gamma_{2} r\right)}{\left(\gamma_{2} r\right)^{2}} b_{1}\left[1+\frac{1}{\gamma_{2} r}\right] \sin \theta \cos \phi
$$

$$
\begin{array}{r}
E_{\theta}^{r}=\frac{i \omega \mu_{0}}{\gamma_{2}} H_{y}\left(z_{0}\right) \frac{3 \exp \left(-\gamma_{2} r\right)}{2 \gamma_{2} r}\left\{-a_{1}\left[1+\frac{1}{\gamma_{2} r}\right]\right. \\
\left.-b_{1}\left[1+\frac{1}{\gamma_{2} r}+\frac{1}{\left(\gamma_{2} r\right)^{2}}\right] \cos \theta\right\} \cos \phi
\end{array}
$$




$$
\begin{gathered}
E_{\phi}^{r}=\frac{i \omega \mu_{0}}{\gamma_{2}} H_{y}\left(z_{0}\right) \frac{3 \exp \left(-\gamma_{2} r\right)}{2 \gamma_{2} r}\left\{a_{1}\left[1+\frac{1}{\gamma_{2} r}\right] \cos \theta\right. \\
\left.+b_{1}\left[1+\frac{1}{\gamma_{2} r}+\frac{1}{\left(\gamma_{2} r\right)^{2}}\right]\right\} \sin \phi \\
H_{\tau}^{r}=H_{y}\left(z_{0}\right) \frac{3 \exp \left(-\gamma_{2} r\right)}{\left(\gamma_{2} r\right)^{2}} a_{1}\left[1+\frac{1}{\gamma_{2} r}\right] \sin \theta \sin \phi \quad(19) \\
H_{\theta}^{r}=H_{v}\left(z_{0}\right) \frac{3 \exp \left(-\gamma_{2} r\right)}{2 \gamma_{2} r}\left\{-a_{1}\left[1+\frac{1}{\gamma_{2} r}+\frac{1}{\left(\gamma_{2} r\right)^{2}}\right] \cos \theta\right. \\
\left.H_{\phi}^{r}=H_{y}\left(z_{0}\right) \frac{3 \exp \left(-\gamma_{2} r\right)}{2 \gamma_{2} r}\left\{1+\frac{1}{\gamma_{2} r}\right]\right\} \sin \phi \\
-b_{1}\left[1+\frac{1}{\gamma_{2} r}+\frac{1}{\left(\gamma_{2} r\right)^{2}}\right]
\end{gathered}
$$

where $a_{1}$ and $b_{1}$ are given by (9.25.10) and (11) of Stratton [1941]. If $\left|\gamma_{2} a\right|<<1$ and $\left.\sigma_{c}\right\rangle>\sigma, a_{1}$ and $b_{1}$ are approximated by

$$
\begin{gathered}
a_{1}=-\frac{\left(\gamma_{2} a\right)^{3}}{3}\left[1+\frac{3}{5}\left(\gamma_{2} a\right)^{2}-3 \frac{\mu}{\mu_{0} \gamma_{3} a}+3\left(\frac{\mu}{\mu_{0} \gamma_{3} a}\right)^{2}+\ldots\right] \\
b_{1}=\frac{2}{3}\left(\gamma_{2} a\right)^{3}\left[1-\frac{3}{10}\left(\gamma_{2} a\right)^{2}-\frac{\sigma}{\sigma_{c}}\left(\gamma_{3} a\right)+\ldots\right]
\end{gathered}
$$

where $\gamma_{3}=\sqrt{i \omega \mu \sigma_{c}}$ and where the last term of (23) is usually negligible. In the limit of a highly conducting small sphere these approximations result in

$$
a_{1}=-b_{1} / 2=-\left(\gamma_{2} a\right)^{3} / 3 \text {. }
$$

The field components proportional to $b_{1}$ are identical to those of $a \xi$-directed electric dipole [Stratton, 1941; Wait, 1960a] of a dipole moment

$$
\begin{aligned}
I d \xi & =6 \pi E_{x}\left(z_{0}\right) b_{1}\left(\sigma_{2}+i \omega \epsilon_{2}\right) / \gamma_{2}^{3} \\
& =6 \pi H_{y}\left(z_{0}\right) b_{1} / \gamma_{2}^{2}
\end{aligned}
$$

The field components proportional $a_{1}$ are identical to those of an $\eta$-directed magnetic dipole [Wait, 1960a] of a dipole moment

$$
K d \eta=6 \pi i \omega \mu_{0} H_{y}\left(z_{0}\right) a_{1} / \gamma_{2}^{3} .
$$

The electric dipole moment $I d \xi$ of (25) can be attributed to the incident electric field, while the magnetic dipole moment $K d \eta$ of (26) is thought to be caused by the incident magnetic field [Wait, 1950]. This separation of the effects of the electric and magnetic fields is particularly useful if the sphere is excited by a non-plane wave.

The expressions (16) to (21) will apply as long as the local fields are not perturbed by surface reflections.
However, the surface reflections must be considered when computing the scattered fields in the vicinity of the interface between media 1 and 2 .

\subsection{Multiple Reflections Between the Sphere and the Surface}

The fields in the vicinity of the sphere may be perturbed by surface reflections. If the radius of the sphere $a$ is small relative to the skin depth of the dissipative medium $\delta=\sqrt{2 /\left(\omega \mu_{0} \sigma\right)}$, the effect of these second order reflections can be shown to be small.

The $E$ and $I I$ fields of (16) to (21) will be reflected downwards from the interface between the media 1 and 2 , but only the field components which leave the sphere at an angle $\theta \approx \pi$ arrive back into the vicinity of the sphere. At this angle $E_{r 2}^{r}=H_{r 2}^{r}=0$, the $E$ field is in the $x$ direction and the $H$ field is in the $y$ direction. If $\left|\gamma_{2}\right|>>k$, the field incident from the sphere $E_{x i}$ and $H_{y i}$ and the field reflected from the surface $E_{x r}$ and $H_{y r}$ are related at the surface by

$$
\begin{gathered}
E_{x r} \approx E_{x i} \\
H_{y r} \approx-H_{y i} .
\end{gathered}
$$

The field components $E_{x r}\left(z_{0}\right)$ and $H_{y r}\left(z_{0}\right)$, which are reflected from the interface into the vicinity of the sphere, may be related to the field component $E_{x}\left(z_{0}\right)$ and $H_{y}\left(z_{0}\right)$ of the plane wave which excited the sphere originally. Equations (17), (18), (20), $(21),(27)$, and (28) result in

$$
\begin{aligned}
\frac{E_{x r}\left(z_{0}\right)}{E_{x}\left(z_{0}\right)}= & \frac{3}{2} \exp \left(-2 \gamma_{2} z_{0}\right) \\
& \left\{\left(b_{1}-a_{1}\right)\left[\frac{1}{2 \gamma_{2} z_{0}}+\frac{1}{\left(2 \gamma_{2} z_{0}\right)^{2}}\right]+\frac{b_{1}}{\left(2 \gamma_{2} z_{0}\right)^{3}}\right\} \\
\frac{H_{y r}\left(z_{0}\right)}{H_{y}\left(z_{0}\right)}= & \frac{3}{2} \exp \left(-2 \gamma_{2} z_{0}\right) \\
& \left\{\left(b_{1}-a_{1}\right)\left[\frac{1}{2 \gamma_{2} z_{0}}+\frac{1}{\left(2 \gamma_{2} z_{0}\right)^{2}}\right]-\frac{a_{1}}{\left(2 \gamma_{2} z_{0}\right)^{3}}\right\} .
\end{aligned}
$$

The ratios (29) and (30) apply also to amplitudes of successively higher order reflections in the vicinity of the sphere. When accounting for the higher order reflections, the net fields incident on the sphere are given by

$$
\begin{aligned}
& \left.E_{x}\left(z_{0}\right)\right|_{\text {Total }}=E_{x}\left(z_{0}\right) \sum_{n=0}^{\infty}\left[\frac{E_{x r}\left(z_{0}\right)}{E_{x}\left(z_{0}\right)}\right]^{n} \\
& \left.H_{y}\left(z_{0}\right)\right|_{\text {Tota } 1}=H_{y}\left(z_{0}\right) \sum_{n=0}^{\infty}\left[\frac{H_{y r}\left(z_{0}\right)}{H_{y}\left(z_{0}\right)}\right]^{n} .
\end{aligned}
$$

The effective dipole moment of the sphere is related to the total incident field in the same way it was related to the field component of the plane wave in (25) and (26). These effective dipole moments of the sphere are obtained by substituting (31) and (32) for $E_{x}\left(z_{0}\right)$ and $H_{y}\left(z_{0}\right)$ in $(25)$ and $(26)$. The effective dipole moment can be expressed as

$$
I d \xi=H_{y}\left(z_{0}\right) F_{e} \sum_{n=0}^{\infty} G_{e}^{n}
$$




$$
K d \eta=H_{y}\left(z_{0}\right) F_{m} \sum_{n=0}^{\infty} G_{m}^{n}
$$

where

$$
\begin{gathered}
F_{e}=6 \pi b_{1} / \gamma_{2}^{2} \\
\approx 4 \pi \gamma_{2} a^{3} \\
F_{m}=6 \pi i \omega \mu_{0} a_{1} / \gamma_{2}^{3} \\
\approx-2 \pi i \omega \mu_{0} a^{3}
\end{gathered}
$$

$G_{i}=\left(\gamma_{2} a\right)^{2} \frac{a}{2 z_{0}} \exp \left(-2 \gamma_{2} z_{0}\right)$

$$
\begin{aligned}
& \left\{\frac{3}{2}\left[1+\frac{1}{2 \gamma_{2} z_{0}}\right]+\frac{c_{i}}{\left(2 \gamma_{2} z_{0}\right)^{2}}\right\} \\
& c_{e}=1 \\
& c_{m}=1 / 2 .
\end{aligned}
$$

The series (33) and (34) are rapidly convergent for $a$ $\langle\delta=\sqrt{2} /|\gamma|$, even if the exponential factor of (37) is of the order of unity. It follows from section 2.1 that the variation of the incident field over the volume of the sphere may be neglected if $a$ is small relative to $\delta$. The neglect of multiple reflections between the sphere and the surface is therefore permissible $\left(\left|G_{i}\right|<<1\right)$, if the field variation is small over the volume of the sphere, as it has been assumed in deriving the field components of section 3.1.

\subsection{Fields of the Subsurface Horizontal Electric and Magnetic Dipoles}

The fields of a subsurface horizontal electric dipole can be obtained from the results of Wait [1961]. The time rate of change of the magnetic induction is approximated for $\left|\gamma_{2} \rho\right|>>1$ by

$$
\dot{B}_{\phi}^{h e}=\frac{i \omega \mu_{0} I d \xi}{2 \pi \rho^{3} \gamma_{2}}\left[1+i k \rho+(i k \rho)^{2}\right] e^{-i k_{\rho}-\gamma_{2} z_{0}} \cos \phi,
$$

which is numerically equal to the voltage induced in a vertical loop of $1 \mathrm{~m}^{2}$ area.

The fields $\dot{B}_{\phi}^{h m}$ produced in the lossless medium by the subsurface horizontal magnetic dipole can be related to the subsurface fields of a vertical electric (superscript ve) and a horizontal electric (superscript he) dipole that are located in medium 1 . It has been shown by Galejs [1961] that

$$
E_{z}^{h m}\left(z_{s}=z_{0}, z_{r}==0\right)=\operatorname{css} \phi H_{\phi^{\prime}}^{v e}\left(z_{s}^{\prime}=0, z_{r}^{\prime}=z_{0}\right) \frac{K d \eta}{I d z^{\prime}}
$$

In the above equation the subscripts $s$ and $r$ refer to source and receiver coordinates respectively. The horizontal magnetic dipole is oriented in the $y$ direction. The unprimed coordinates are defined as in figure $1 ; \phi^{\prime}$ and $\rho^{\prime}$ coordinates are defined in a cylindrical system, centered in that location of the air- conductor interface, where the fields of the subsurface horizontal magnetic dipole should be detected, and with the $\phi^{\prime}=0$ direction parallel to the $x$-axis. With $H_{\phi^{\prime}}^{v e}\left(z_{s}^{\prime}=0, \quad z_{r}^{\prime}=z_{0}\right)$ given by (11), it follows from (41) that

$$
E_{z}^{h m}=\frac{i k K d \eta}{2 \rho \pi}\left(1+\frac{1}{i k \rho}\right) e^{-i k_{\rho}-\gamma_{2} z_{0}} \cos \phi .
$$

The electric field $E_{\rho}^{h m}$ is related to $H_{\phi}^{\text {he }}$ by (13) where $H_{\phi}^{h e}$ is obtained from (4), (21), (22), (27), and (28) of Wait [1961]. This gives

$$
\begin{array}{r}
E_{\rho}^{h m}=\frac{K d \eta}{2 \pi \gamma_{2} \rho^{3}}\left\{(1+i k \rho)\left(1-\gamma_{2} z\right)-\left(1+\frac{k^{2} z}{\gamma_{2}}\right)[2+2 i k \rho\right. \\
\left.\left.+(i k \rho)^{2}\right]\right\} e^{-i k_{\rho}-\gamma_{2} z_{0}} \cos \phi \cdot
\end{array}
$$

The corresponding time rate of change of the magnetic induction is

$$
\begin{aligned}
\dot{B}_{\phi}^{h m} & =\frac{\partial E_{z}^{h m}}{\partial \rho}-\frac{\partial E_{\rho}^{h m}}{\partial z} \\
& =-\frac{K d \eta}{2 \pi \rho^{3}}\left[1+(i k \rho)+(i k \rho)^{2}\right] e^{-i k_{\rho}-\gamma_{2} z_{0}} \cos \phi .
\end{aligned}
$$

The combined surface fields are obtained by adding (40) and (44). After assuming that the terms proportional to $(i k \rho)^{2}$ are negligible it follows that

$$
\dot{B}_{\phi} \approx-\frac{(K d \eta)^{\prime}}{2 \pi \rho^{3}}(1+i k \rho) e^{-i k_{\rho}-\gamma_{2} z_{0}} \cos \phi .
$$

The equivalent magnetic dipole moment $(K d \eta)^{\prime}$ is defined as

$$
(K d \eta)^{\prime}=K d \eta-\frac{i \omega \mu_{0} I d \xi}{\gamma_{2}}
$$

After applying (33) to $(36),(K d \eta)^{\prime}$ becomes

$$
(K d \eta)^{\prime}=-\frac{6 \pi i \omega \mu_{0} H_{y}\left(z_{0}\right)}{\gamma_{2}^{3}}\left[b_{1} \sum_{n=0}^{\infty} G_{e}^{n}-a_{1} \sum_{n=0}^{\infty} G_{m}^{n}\right] .
$$

If it is permissible to ignore the multiple reflections and to apply the approximation $(24),(K d \eta)^{\prime}$ simplifies to

$$
\begin{gathered}
(K d \eta)^{\prime}=18 \pi i \omega \mu_{0} H_{y}\left(z_{0}\right) a_{1} / \gamma_{2}^{3} \\
=-6 \pi i \omega \mu_{0} a^{3} H_{y}\left(z_{0}\right) .
\end{gathered}
$$

This is three times the magnetic dipole moment in (26), which was due to the incident magnetic field only.

\section{Response to a Sinusoidal Signal}

The overall response will be calculated for excitations by a plane surface wave, by vertical electric and horizontal magnetic dipoles. For a plane surface 
wave (superscript $p l$ ) excitation (5), (22), (23), (45), and (47) give

$$
\dot{B}_{\phi}^{p l}=\frac{H_{0}(\omega) a^{3} i \omega \mu_{0}}{\rho^{3}}[1+i k \rho]\left[\sum_{n=0}^{\infty} F_{n}\right] e^{-i k_{\rho}-2 \gamma_{2} z_{0}} \cos \phi
$$

where the $H$ vector of the plane wave is in the $y$-direction and the $\rho$ and $\phi$ coordinates of the observation point are arbitrary. For a vertical electric dipole excitation (11), (22), (23), (45), and (47) result in

$$
\dot{B}_{\phi}^{v e}=\frac{I(\omega) d s a^{3} \dot{i} \omega \mu_{0}}{2 \pi \rho^{5}}[1+2 i k \rho]\left[\sum_{n=0}^{\infty} F_{n}\right] e^{-2 i k_{\rho}-2 \gamma_{2} z_{0}},
$$

where both the dipole and the observation point are located at the same point in the $y=0$ plane. For a horizontal magnetic dipole (or a vertical loop of area $d a$ ) excitation (14), (22), (23), (45), and (47) result in

$$
\dot{B}_{\phi}^{h m}=\frac{I(\omega) d a a^{3} i \omega \mu_{0}}{2 \pi \rho^{6}}[1+2 i k \rho]\left[\sum_{n=0}^{\infty} F_{n}\right] e^{-2 i k_{\rho}-2 \gamma_{2} z_{0}} .
$$

In the above expression $F_{n}$ is given by

$$
\begin{array}{r}
F_{n}=2\left[1-\frac{3}{10}\left(\gamma_{2} a\right)^{2}+\ldots\right] G_{e}^{n}+\left[1+\frac{3}{5}\left(\gamma_{2} a\right)^{2}\right. \\
\left.-3 \frac{\mu}{\mu_{0} \gamma_{3} a}+3\left(\frac{\mu}{\mu_{0} \gamma_{3} a}\right)^{2}+\ldots\right] G_{m}^{n},
\end{array}
$$

where $G_{i}$ with $i=e$ or $m$ is defined by (37) to (39). The summation over $n$ may be approximated by 3 when neglecting multiple reflections between the small sized, highly conducting sphere and the interface between media 1 and 2 . The attenuation $\alpha$ (in $d b)$ of $\dot{B}_{\phi}\left(z=z_{0}\right)$ relative to $\dot{B}_{\phi}(z=0)$, which is due to the exponential factors in (49) to (51) is given by

$$
\begin{aligned}
\alpha & =20 \log _{10}\left|e^{-2 \gamma_{2} z_{0}}\right| \\
& =8.68 \times 2 \frac{z_{0}}{\delta} .
\end{aligned}
$$

For $\rho<<\lambda, k \rho<<1$ and the three expressions of $\dot{B}_{\phi}$ are related as

$$
\left|\dot{B}_{\phi}^{p 1}\right|:\left|\dot{B}_{\phi}^{v e}\right|:\left|\dot{B}_{\phi}^{h m}\right|=H_{0}(\omega): \frac{I(\omega) d s}{2 \pi \rho^{2}}: \frac{I(\omega) d a}{2 \pi \rho^{3}} .
$$

The above $\dot{R}_{\phi}$ figures are related in the same way as the surface fields $H_{0}(\omega), H_{\phi}^{v e}$ and $H_{\phi}^{h m}$ of (5), (11), and (14). The magnitude of the leading term of (51) may be written as

$$
\left|\dot{B}_{\phi}^{h m}\right| \approx \frac{3 I(\omega) d a}{\pi \rho^{6} \sigma} \frac{a^{3}}{\delta^{2}} \exp \left(-2 \frac{z_{0}}{\delta}\right)
$$

In a numerical example, the magnitude of $\dot{B}_{\phi}^{h m}$ will be calculated using the following parameters: $a=2.5 \mathrm{~m}, \delta=25 \mathrm{~m}, z_{0}=12.5 \mathrm{~m}, \rho=10^{2} \mathrm{~m}, \sigma=10^{-3}$ $\mathrm{mho} / \mathrm{m}$ (dry ground), and $I(\omega) d a=10^{6} \mathrm{Am}^{2}$. It follows that $\left|\dot{B}_{\phi}^{h m}\right|=9 \times 10^{-6} \mathrm{~V} / \mathrm{m}^{2}$.
The response of the buried spheres may be simply related to the response of the spheres in free space. The leading term of the near field response of the buried sphere in (49), (50), or (51) is of the form $\left|\dot{B}_{\phi}\right|=A \exp \left(-2 z_{0} / \delta\right)$. The response of the same sphere in free space is $\left|\dot{B}_{\phi}\right|=A / m$, where $m=12$ for identical dipole excitations and $m=6$ if the former surface excitation $H_{0}(\omega)$ is applied directly to the sphere. The increase in the scattering cross section of the sphere in the dissipative medium is therefore sufficient to offset the refraction losses for sufficiently shallow depths $z_{0}$.

\section{Transient Response}

The overall transient response will be calculated for an impulse-type plane wave surface excitation $H_{0} T \delta(t)$, for a step of voltage $V$ applied to the electric dipole of capacity $C$ and an impulse of current $I_{0} T \delta(t)$ applied to the magnetic dipole. This results with neglected multiple reflections in

$$
\begin{aligned}
\dot{B}_{\phi}(i \omega)=i \omega D[1+i \omega p & +q(i \omega)^{-0.5} \\
& \left.+q^{2}(i \omega)^{-1}+\ldots\right] e^{-i \omega h-\sqrt{i \omega g}}
\end{aligned}
$$

where

$$
q=-\frac{\mu}{\mu_{0} \sqrt{\mu \sigma_{c} \alpha}}
$$

and

$$
\sqrt{g}=2 \sqrt{\mu_{0} \sigma} z_{0} .
$$

The constants $D, p$, and $h$ have the following values for the three types of excitations considered:

\begin{tabular}{l|c|c|c}
\hline \hline & Plane wave & Electric dipole & Magnetic dipole \\
\hline$D$ & $\frac{H_{0} T 3 a^{3} \mu_{0} \cos \phi}{\rho^{3}}$ & $\frac{3 C T d s a^{3} \mu_{9}}{2 \pi \rho^{5}}$ & $\frac{3 I_{0} T d a a^{3} \mu_{9}}{2 \pi \rho^{6}}$ \\
$p$ & $(\rho / c)$ & $(2 \rho / c)$ & $(2 \rho / c)$ \\
$h$ & $\rho / c$ & $2 \rho / c$ & $2 \rho / c$ \\
\hline
\end{tabular}

The transient response is calculated with the aid of the Fourier transform pairs (208), (802), (806), and (801) of Campbell and Foster [1948]. This results in

$$
\begin{aligned}
\dot{B}_{\phi}(\tau)=\frac{8 D}{\sqrt{\pi} g^{2}} e^{-y} y^{1.5} & {\left[\left(4 y^{4}-20 y^{3}+15 y^{2}\right) \frac{2 p}{g}\right.} \\
& \left.+2 y^{2}-y\left(3+2 q_{1}\right)+2 q_{1}^{2}+q_{1}\right],
\end{aligned}
$$

where

$$
\begin{gathered}
\tau=t-h \\
y=\frac{g}{4 \tau} \\
q_{1}=-\frac{q \sqrt{g}}{2}=\sqrt{\frac{\mu \sigma}{\mu_{0} \sigma_{c}}} \frac{z_{0}}{a}
\end{gathered}
$$




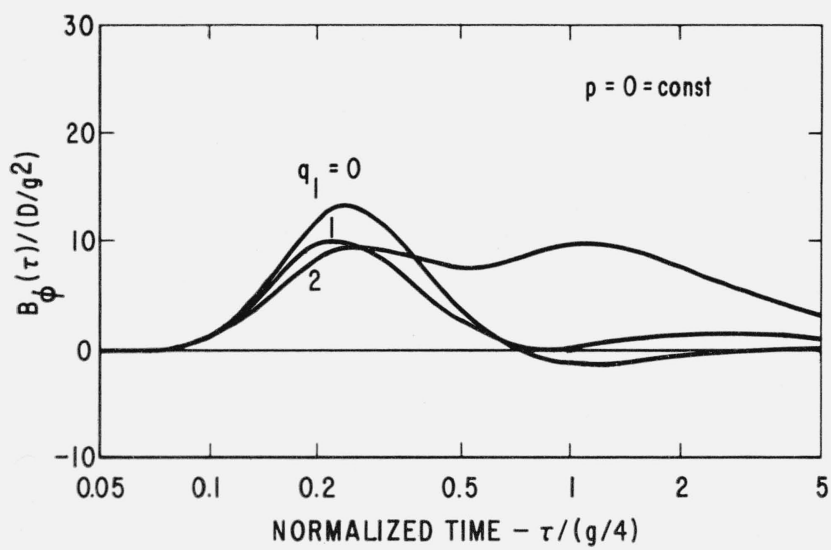

FIGURE 2. Transient response for $\mathrm{p}=\mathbf{0}$.

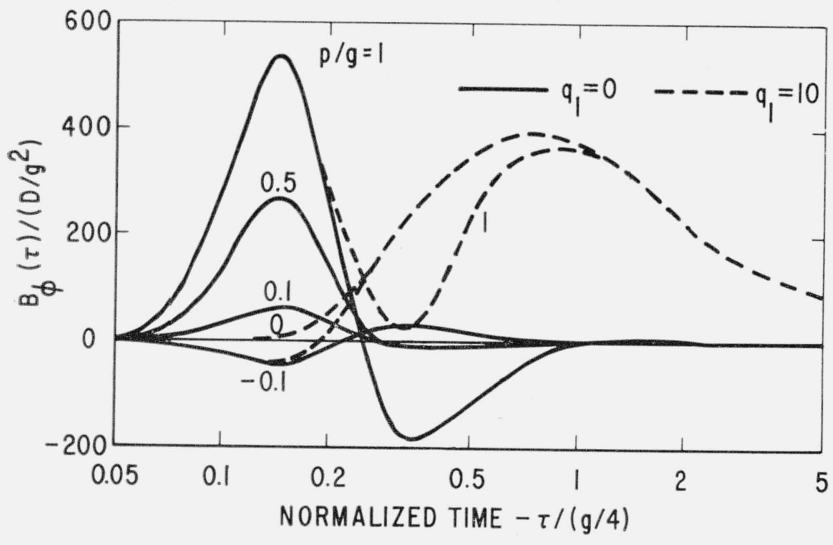

FiguRE 3. Transient response for $\mathrm{p} \neq 0$.

The response (51) has been plotted in figures 2 and 3 for several values of the parameters $(p / g)$ and $q_{1}$. The $p \neq 0, q_{1} \neq 0$ curves differ insignificantly from the $p \neq 0, q_{1}=0$ curves, unless $q_{1}>>1$. If the terms proportional to $(p / g)$ and to $q_{1}$ are negligible, this response exhibits a minimum of

$$
\left.\dot{B}_{\phi}(\tau)\right|_{\min }=-1.69 D / g^{2}
$$

for $y=0.92$, it is zero for $y=1.5$, and it exhibits a maximum

$$
\left.\dot{B}_{\phi}(\tau)\right|_{\max }=13.2 D / g^{2}
$$

for $y_{\max }=4.08$.

The following parameters will be considered by the way of a numerical example: $a=2.5 \mathrm{~m}, z_{0}=12.5 \mathrm{~m}$, $\rho=100 \mathrm{~m}, \sigma=10^{-3} \mathrm{mho} / \mathrm{m}, \sigma_{c}=10^{7} \mathrm{mho} / \mathrm{m}, \mu / \mu_{0}=10^{3}$, and a horizontal magnetic dipole of $I_{0} d a=10^{6} \mathrm{~A} / \mathrm{m}^{2}$. It follows that $q_{1}=2.3 \times 10^{-3}$ and $p / g=0.85$. This response exhibits a maximum value of $\left.\dot{B}_{\phi}^{h m}(\tau)\right|_{\max }$ $=430 D / g^{2}$. This is equal to $64 \times 10^{-6} \mathrm{~V} / \mathrm{m}^{2}$ if $T=10^{-7}$ sec.

\section{Discussion}

In the preceding calculations, the semi-infinite lossy medium was assumed to be excited by a plane surface wave or by a vertical electric and horizontal magnetic dipole that are located in the interface between two media. Modification of the model of analysis by buried transmitters and receivers offers no further advantages. For horizontal distances $\rho$ much larger than the depth under consideration $z_{0}$, the transmitted energy propagates above the lossy medium and penetrates vertically downward from the interface. A horizontal magnetic dipole located in the lossy medium would be coupled inefficiently to the surface wave. (A horizontal electric dipole is inefficient when its height above the ground is much less than $\lambda$. Its efficiency is only slightly decreased for shallow burial depths $z_{0}$, where $z_{0}<\delta=$ skin depth.) Because of the continuity of the tangential $H$ field across the interface, a vertical pick-up loop would be as sensitive below as above the interface. A buried receiver would offer advantages only if it would be possible to isolate the wave incident from the reflecting object on the interface and the wave reflected by the interface. However, even this may have merits only in the direct vicinity of the reflecting object $\left(z_{0}>\rho\right)$.

\section{References}

Baños, A., and Wesley, J. P., The horizontal electric dipole in a conducting half-space, Part I, Univ. Calif. Marine Phys. Lab., Scripps Inst. Oceanography, SIO Ref. 53-33 (Sept. 1953); Part II, SIO Ref. 54-31 (Äug. 1954).

Campbell, G. A., Foster, R. M., Fourier integrals for practical applications, (D. Van Nostrand Company, New York, 1948).

Galejs, J., Impulse excitation of a conducting medium, IRE Trans. Ant. Prop. AP-8, 227-228 (Mar. 1960).

Galejs, J., Excitation of VLF and ELF radio waves by a horizontal magnetic dipole, J. Research NBS 65D (Radio Prop.) 305-311 (May-June 1961).

Keilson, J., and Row, R., Transfer of transient electromagnetic surface waves into a lossy medium, J. Appl. Phys. 30, 1595-1598 (Oct. 1959).

Norton, K. A., The propagation of radio waves over the surface of the earth and in the upper atmosphere, Part I, Proc. IRE, 24, 1367-1387, (Oct. 1936); Part II, Proc. IRE, 25, 1203-1236 (Sept. 1937).

Norton, K. A., The physical reality of space and surface waves in the radiation field of radio antennas, Proc. IRE 25, 1192-1202 (Sept. 1937).

Richards, P. I., Transients in conducting media, IRE Trans. Ant. Prop. AP-6, 178-182 (Apr. 1958).

Sommerfeld, A., Partial differential equations in physics, Ch. VI (Academic Press Inc., New York, 1949).

Stratton, J. A., Electromagnetic theory, Sections 9.28-9.31 (McGraw-Hill Book Co., New York, 1941).

Wait, J. R., Electromagnetic radiation in the earth, Ph. D. thesis, University of Toronto, 1950.

Wait, J. R., A conducting sphere in a time varying magnetic, J. Geophys. 16, 666-672 (Oct. 1951).

Wait, J. R., On the electromagnetic response of a conducting shpere of a dipole field, J. Geophys. 25, 649-658 (June 1960a).

Wait, J. R., Terrestrial propagation of VLF radio wavesa theoretical investigation, J. Research NBS 64D (Radio Prop.) 153-203 (Mar.-Apr. 1960b).

Wait, J. R., Propagation of electromagnetic pulses in a homogeneous conducting earth, App. Sci. Research B8, 213-253 (1960c).

Wait, J. R., The electromagnetic fields of a horizontal dipole in the presence of a conducting half-space, Can. J. Phys. 39, 1017-1028 (1961).

(Paper 66D5-221) 